\title{
Estratégia como prática social: sistematização das definições e conceitos
}

\author{
Nágila Giovanna Silva Vilela ${ }^{1}$ \\ Ronaldo de Oliveira Santos Jbunior ${ }^{2}$
}

\begin{abstract}
Resumo: $\mathrm{O}$ interesse em estudos na seara da Estratégia como Prática Social apresenta crescimento acelerado à medida em que atrai a curiosidade de pesquisadores atuantes em estudos estratégicos e organizacionais. $\mathrm{Na}$ temática, questões relacionadas aos indivíduos, suas emoções e motivações, são levadas em consideração de maneira mais evidente do que a percebida em pesquisas tradicionais em estratégia. Trata-se, portanto, de uma abordagem interpretativista em que o mundo não pode ser entendido de forma independente dos atores sociais e os processos que o produzem. Dessa maneira, este artigo, caracterizado como ensaio teórico, tem como objetivo oferecer uma sistematização das definições e conceitos da proposta da Estratégia como Prática Social. Diante das reflexões apresentadas no texto, busca-se contribuir para essa literatura em formação com a revisão de seus conceitos basilares desde os estudos seminais até o contexto contemporâneo de estudos em estratégia e organizações.
\end{abstract}

Palavras-chave: Estratégia como prática. Prática social. Ensaio teórico.

Abstract: The interest in studies in the area of Strategy as Social Practice presents accelerated growth as it attracts the curiosity of researchers working in strategic and organizational studies. In the topic, issues related to individuals, their emotions and motivations, are taken into account in a more evident way than is perceived in traditional strategy research, so it is an interpretative approach in which the world can not be understood in a way independent of the social actors and the processes that produce it. In this way, this article aims to offer a systematization of the definitions and concepts of the Strategy as a Practice and is characterized as a theoretical essay. In the light of the reflections presented in the text, we seek to contribute to this literature in formation with the revision of its basic concepts from its seminal studies to the contemporary context of research in strategy and organizations.

Palavras-chave: Strategy as practice. Social practice. Theoretical essay.

\section{Introdução}

O campo da estratégia como prática tem crescido rapidamente e associa-se à virada prática, uma vez que foi nesse cenário que se desenvolveu. A virada prática da estratégia data de 2001, em Bruxelas, na Bélgica, quando o European Institute for Advanced Studies in Management realizou um encontro de pesquisadores da área de estratégia (MACIEL; AUGUSTO, 2013). Nesse momento surgiu a abordagem da estratégia como prática, que já tinha sido sugerida por Whittington cinco anos antes (MACIEL;

\footnotetext{
${ }^{1}$ Doutoranda em Administração pela Universidade de São Paulo. Membro dos grupos de pesquisa: Estratégia, Dinâmica e Comportamento em Organizações - Processos e Práticas; Autoconhecimento e Desenvolvimento de Carreira. E-mail: nagilavilela@gmail.com.

${ }^{2}$ Doutorando em Administração pela Universidade de São Paulo. Membro dos grupos de pesquisa: Estratégia e Processo Decisório. Stakeholders \& Networks.E-mail: ronaldojhr@gmail.com.
}

Página 57 Caderno de Ciências Sociais Aplicadas, Vitória da Conquista/BA, vol. 15, n 25, ano 15, p. 57-73, jan/jun 2018. 
AUGUSTO, 2013). Essa perspectiva europeia foi uma forma alternativa de pensar estratégia no campo de estudos da estratégia, que até então tinha predominância do pensamento norte-americano (MACIEL; AUGUSTO, 2013). Trata-se, portanto, de um movimento europeu oriundo da teoria social dos anos 1980 em que se acrescenta um caráter sociológico para a estratégia ao mesmo tempo que torna mais amplo os atores compreendidos na estratégia (DIAS; ROSSETO; MARINHO, 2017).

No cerne da estratégia como prática estava a promessa da virada prática, que ressaltava a ação dos estrategistas em vez de considerar que a estratégia era mais uma propriedade das organizações (MACIEL; AUGUSTO, 2013). Assim, enquanto a estratégia parece ter se esquecido do ser humano, a estratégia como prática social dá importância para a efetivação da estratégia, por quem e como é realizada, o que é utilizado e as implicações na formulação da estratégia (JARZABKOWSKI; SPEE, 2009).

Sob essa perspectiva há uma preocupação com os indivíduos, suas emoções e motivações, algo que aparentemente havia sido perdido na pesquisa em estratégia (JARZABKOWSKI; SPEE, 2009). Trata-se, portanto, de uma “[...] abordagem interpretativista em que o mundo não pode ser entendido de forma independente dos atores sociais e os processos que o produzem" (JARZABKOWSKI; KAPLAN, 2010, p. 55).

O crescimento do campo da estratégia como prática social é notório pelos números de artigos e livros que tratam do fenômeno (MACIEL; AUGUSTO, 2013). Journals como Organization Studies e Strategic Organization recebem um grande número de publicações dessa temática e o Journal of Management Studies e Human Relations inclusive trouxeram edições especiais para estratégia como prática (MACIEL; AUGUSTO, 2013). Na literatura brasileira, apesar de os estudos sobre a temática ainda não terem sido completamente difundidos, o assunto tem recebido atenção dos acadêmicos da área (ZWICK; SILVA; BRITO, 2014).

Diante desse contexto, o objetivo do presente artigo é oferecer uma sistematização das definições e conceitos da proposta de Estratégia como Prática. O interesse nesse tópico está relacionado ao crescimento de publicações acadêmicas a respeito dessa temática, apesar de, segundo Jarzabkowski e Kaplan (2010), ainda levar tempo para que a estratégia como prática social seja consolidada no campo da gestão estratégica.

Em termos metodológicos, esse artigo é qualificado como um ensaio teórico, caracterizado “[...] pela sua natureza reflexiva e interpretativa” (MENEGHETTI, 2011, p. 322). Buscou-se sistematizar os conceitos a respeito da gestão estratégica, da prática social, bem como de práxis, praticantes, práticas e

Página 58 Caderno de Ciências Sociais Aplicadas, Vitória da Conquista/BA, vol. 15, n 25, ano 15, p. 57-73, jan/jun 2018. 
strategizing - estrutura de conceituação adotada pelos estudiosos de estratégia (JARZABKOWSKI; SPEE; SMETS, 2013). Para tanto, a pesquisa bibliográfica foi realizada nos principais livros, periódicos e journals (DOXSEY; DE RIZ, 2003) da área de estratégia. Ao final do artigo são apresentadas as considerações finais do trabalho, as contribuições, limitações e os cuidados que devem ser tomados em estudos e investigações futuras de estratégia como prática.

\section{Gestão estratégica}

Os pesquisadores da gestão estratégica atribuem seu surgimento como campo de conhecimento acadêmico ao início dos anos 1960 (RONDA-PUPO; GUERRAS-MARTIN, 2012). Nag, Hambrick e Chen (2007) consideram que os estudos sobre a temática se intensificaram a partir de 1979, quando Schendel e Hofer (1979 apud NAG; HAMBRICK; CHEN, 2007) deram ao campo da política empresarial o nome de gestão estratégica e sugeriram um novo paradigma fundamentado no conceito de estratégia. A partir de então surgiram diferentes definições para estratégia, colaborando para a fragilidade e falta de consenso para o termo (NAG; HAMBRICK; CHEN, 2007).

Uma definição de estratégia foi estabelecida por Chandler (1962). Ao relacionar estratégia e estrutura, o autor definiu o primeiro como o delineamento do crescimento, isto é, o plano de alocação dos recursos compatível com a demanda esperada. A estrutura, por sua vez, refere-se à divisão de atividades e recursos, de forma que os recursos atuais da empresa sejam integrados à demanda. Nesse sentido, o crescimento estratégico está relacionado com a habilidade de utilizar os recursos existentes ou expandir com maior aproveitamento.

Porter, por sua vez definiu a estratégia como “[...] a criação de uma posição ímpar e valiosa, envolvendo um conjunto diverso de atividades” (PORTER, 1996 p. 10). O fundamento é que não há sempre um caminho melhor ou uma estratégia ideal. Se assim o fosse, o sucesso seria da organização que mais rápido descobrisse e se apropriasse da mesma. Dessa forma, o autor considera que o fundamento do posicionamento estratégico consiste em diferenciar-se dos concorrentes.

Mintzberg, Ahlstrand e Lampel (2007) também contribuíram para os estudos sobre estratégia no livro Safári de Estratégia. Levando em consideração a ideia de que a essência da estratégia é um tanto confusa, que ela pode ocorrer em diversos níveis e envolver diversos processos de pensamento, são apresentadas dez escolas que contribuíram para o planejamento estratégico e desenvolvimento da administração estratégica. Essas dez escolas representam concepções distintas para a estratégia, sendo

Página 59 Caderno de Ciências Sociais Aplicadas, Vitória da Conquista/BA, vol. 15, n 25, ano 15, p. 57-73, jan/jun 2018. 
que cada uma atribui uma definição à estratégia, com vantagens e desvantagens, pressupostos e contribuições. Todas as escolas possuem sua parcela de contribuição para a estratégia e algumas inclusive se desenvolveram a partir do conceito definido de escola(s) anterior(es).

Sampaio, Fortunato e Bastos (2013, p. 481), por sua vez, consideram a estratégia como uma sequência de etapas, tais como:

[...] determinar a missão, os valores e a visão de futuro, analisar o ambiente externo e tomar decisões de adequação dos fatores internos, avaliar cenários e, com base em reflexões analíticas e integradas, definir o curso de ações (posturas, objetivos e metas) para atingir a visão almejada.

No campo de estudos da estratégia, novos conceitos e abordagens têm surgido e contribuído para a área, em especial a corrente da estratégia como prática social ou estratégia como prática (ZWICK; SILVA; BRITO, 2014). Esse tópico é abordado a seguir.

\section{Estratégia como prática social}

$\mathrm{Na}$ perspectiva da estratégia como prática, estratégia é entendida como uma atividade socialmente realizada, enquanto que o "fazer estratégia" ou strategiżing compreende aquelas ações, interações e negociações de múltiplos atores e as práticas situadas em que esses se baseiam na realização da atividade (JARZABKOWSKI, 2005). Nessa abordagem, a estratégia não é compreendida como propriedade estática de uma empresa, mas é continuamente criada na realização do trabalho estratégico (JARZABKOWSKI; SPEE; SMETS, 2013).

Whittington (1996) estabeleceu-se como um dos autores pioneiros a enfatizar, de forma explícita, a importância da noção de estratégia como prática social nos estudos de administração estratégica (TURETA; LIMA, 2011). Na ocasião, o autor distinguiu as diferentes perspectivas da estratégia (como plano, política, processo e prática) e afirmou que o foco da última se encontra na estratégia como uma "prática" social, ou seja, em como os praticantes da estratégia realmente atuam e interagem (WHITTINGTON, 1996; TURETA; LIMA, 2011).

No contexto de crescimento em publicações acadêmicas relacionadas ao estudo da prática estratégica, observa-se que a temática foi originada no cenário da chamada Virada Prática, amplo movimento dentro do campo das ciências sociais, que ganhou espaço a partir da década de 1980.

Página 60 Caderno de Ciências Sociais Aplicadas, Vitória da Conquista/BA, vol. 15, n 25, ano 15, p. 57-73, jan/jun 2018. 
Autores como Giddens (1984), Bourdieu (1990), Certeau (1984) e Schatzki (1996) estabeleceram-se como pesquisadores seminais da perspectiva de estudo da estratégia como prática.

De acordo com Whittington (2006), a disciplina de estratégia trata a estratégia como algo pertencente à organização, uma propriedade da mesma. No entanto, gradativamente a estratégia tem sido considerada uma prática, isto é, algo que as pessoas fazem (WHITTINGTON, 2006). Na lógica dessa abordagem, é possível notar o objetivo de compreender a maneira como as pessoas realizam de fato seu trabalho dentro das organizações nas quais estão inseridas. Há também uma preocupação no que se refere à efetividade do desempenho individual dessas pessoas enquanto atores/praticantes que, por sua vez, influencia o desempenho da organização como um todo (WHITTINGTON, 2006).

A estratégia como prática difere-se da pesquisa tradicional de estratégia em sua visão de agência e seu foco na produção e reprodução da ação estratégica ao invés de procurar explicar a mudança estratégica e o desempenho da empresa (JARZABKOWSKI 2005, 2008, WHITTINGTON, 2007; JARZABKOWSKI; SPEE, 2009). Além disso, a abordagem trata da perspectiva estratégica em múltiplos níveis de ação e interação, e não apenas no nível empresarial (JARZABKOWSKI, 2005, 2008; WHITTINGTON, 2007; JARZABKOWSKI; SPEE, 2009). Dessa forma, Whittington (2006) assume a ideia de que a estratégia é uma prática social na qual os praticantes atuam e com a qual interagem. Em outras palavras, a estratégia não é somente algo que as organizações possuem, mas também algo que as pessoas nelas inseridas fazem utilizando o que as organizações possuem, indicando um importante foco naqueles que praticam a estratégia (WHITTINGTON, 2006; ARZABKOWSKI; SPEE, 2009).

Nessa perspectiva, Whittington (2006) afirma que uma organização não seria simplesmente algo atomizado e inserido em uma lógica técnica e institucional, mas que deveria ser entendida como a expressão de princípios sócio estruturais potencialmente diversos. Assim, o autor apresenta um modelo estruturado em três dimensões inter-relacionadas: as práticas, a práxis e os praticantes. Logo, entende-se que as práticas são classificadas como rotinas compartilhadas de atuação ou comportamento que incluem tradições, normas, maneiras de pensar e atitudes em um sentido mais amplo; a práxis, por sua vez, se refere a uma atividade atual que os atores executam na prática; e os praticantes são os atores que realizam atividades de formulação e a implementação da estratégia (WHITTINGTON, 2006).

Em um contexto mais amplo, os praticantes são os indivíduos que elaboram as práticas. Eles moldam a atividade estratégica por meio de quem eles são, como eles agem e quais práticas desenvolvem (JARZABKOWSKI; BALOGUN; SEIDL, 2007). São seres sociais, cujas qualidades 
sócio-políticas, retóricas, culturais e referentes ao sexo moldam a forma como se comportam e o que podem, de fato, alcançar (ROULEAU, 2005; SAMRA-FREDERICKS, 2005; VAARA; WHITTINGTON, 2012).

Por conseguinte, a ideia de práxis diz respeito ao fluxo de atividade em que a estratégia é realizada (JARZABKOWSKI; SPEE, 2009). É um termo para descrever toda a ação humana; é tanto um conceito incorporado que pode ser operacionalizado em diferentes níveis - do micro ao institucional - como dinâmico, deslocando-se fluidamente por meio das interações entre os níveis (JARZABKOWSKI; BALOGUN; SEIDL, 2007).

Por último e seguindo a argumentação, as práticas são ferramentas sociais, simbólicas e materiais, e é por intermédio delas que o trabalho da estratégia é realizado (JARZABKOWSKI; SPEE, 2009).

Jarzabkowski et al. (2013) validam a posição comum no campo da perspectiva de estratégia como prática de que a estratégia não se classifica como uma propriedade estática de uma organização, mas sim como algo criado no contínuo strategizing. Assim, no strategizing estão inseridos todos os tipos de ferramentas para garantir seu acontecimento, incluindo a ideia de rotinas e procedimentos, de recursos discursivos e também artefatos materiais e suas propriedades particulares de influência na atuação dos praticantes.

A unidade de análise para a pesquisa em estratégia como prática são as próprias práticas compostas de atividades recorrentes. Essa definição é ampla e requer que o pesquisador escolha quais atividades acentuar e enfatizar, já que nem toda atividade diária pode ser analisada e teorizada (JARZABKOWSKI; KAPLAN, 2010). As práticas estratégicas podem estar localizadas tanto no nível intra quanto extra organizacional. No nível intraorganizacional, as práticas podem ser específicas da organização, imersas em rotinas, procedimentos operacionais e culturas que moldam o strategizing (NELSON; WINTER, 1982; MARTIN, 2002; WHITTINGTON, 2006). No nível extraorganizacional as práticas derivam de campos sociais ou sistemas abrangentes em que uma organização particular está inserida (WHITTINGTON, 2006) conforme apresentado na Figura 1. Dessa forma, a estratégia como prática fornece conhecimento para além dos processos organizacionais e incorpora atividades estratégicas em um nível mais amplo de práticas da sociedade (WHITTINGTON, 2006, 2007; JARZABKOWSKI; SPEE, 2009).

Página 62 Caderno de Ciências Sociais Aplicadas, Vitória da Conquista/BA, vol. 15, n 25, ano 15, p. 57-73, jan/jun 2018. 


\section{Cadernos de CiênCIas SOCIAIS APLICADAS}

Andriguetto Junior (2016, p. 338) argumenta que:

nessa teoria, o foco de análise está sobre as microatividades dos indivíduos que compõem a organização, como rotinas, práticas estratégicas, reuniões, barganhas, negociações, entre outros. Está centrada nas práxis, práticas e nos praticantes que, em conjunto, fazem a estratégia.

Figura 1: Integração entre práxis, prática e praticantes.

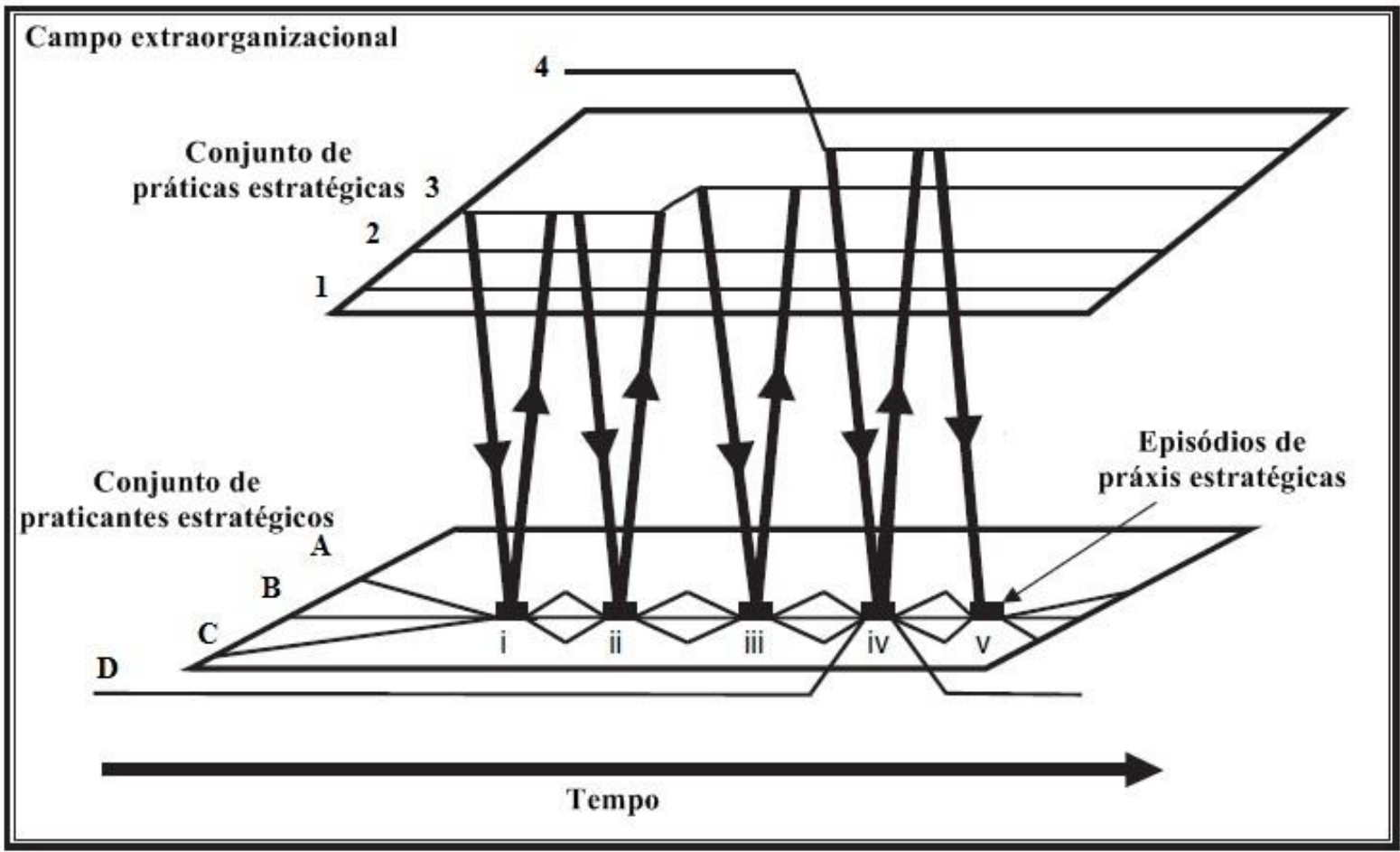

Fonte: Adaptado de Whittington (2006, p. 621)

A estratégia como prática também enfatiza associações entre as perspectivas micro e macro (JARZABKOWSKI, 2004; WHITTINGTON, 2006). Assim, a pesquisa nesse campo pode explicar resultados que são consequentes para a empresa em todos os níveis, desde os menores detalhes do comportamento humano até os níveis institucionais mais amplos, a depender do foco do estudo (JARZABKOWSKI, BALOGUN; SEIDL, 2007). A questão das associações entre micro e macro surgiu porque a agenda de pesquisa de estratégia como prática tem se preocupado em grande parte com o estudo de praticantes, práticas e práxis como microfenômenos (JARZABKOWSKI; SPEE, 2009).

É notório que a Figura 1 reúne práxis, práticas e praticantes dentro de um quadro integrativo de prática estratégica (WHITTINGTON, 2006). Seguindo a teoria da prática, os praticantes são vistos

Página 63 Caderno de Ciências Sociais Aplicadas, Vitória da Conquista/BA, vol. 15, n 25, ano 15, p. 57-73, jan/jun 2018. 
como a conexão crítica entre a práxis intra-organizacional e as práticas organizacionais e extraorganizacionais baseadas nessa práxis (WHITTINGTON, 2006). Nos tópicos a seguir, são abordados os conceitos de práxis, práticas e praticantes para maior compreensão posterior da lógica do strategizing.

\section{a) Práxis}

Práxis é um “[...] termo enfático para descrever toda a ação humana” (RECKWITZ, 2002, p. 249), refere-se ao trabalho da estratégia como rotinas, discursos, conceitos e tecnologias (MARITZ; DU TOIT, 2018). A práxis articula as ações de diferentes indivíduos e grupos e as instituições em que esses indivíduos atuam, sejam elas sociais, econômicas ou políticas (JARZABKOWSKI; BALOGUN; SEIDL, 2007). Em outras palavras, a práxis indica quais as atividades que os praticantes executam na elaboração e implementação da estratégia (WALTER; AUGUSTO; FONSECA, 2011).

A práxis é um conceito que, além de operacionalizado em vários níveis, desde o micro até o institucional, também é dinâmico e pode fluir por meio da interação entre os níveis (JARZABKOWSKI; BALOGUN; SEIDL, 2007).

Os estudos sobre práxis podem se encontrar em três níveis: micro, meso e macro (JARZABKOWSKI; SPEE, 2009). No nível micro estão as pesquisas que analisam a práxis da estratégia em nível individual ou grupal em eventos específicos como uma decisão ou uma reunião. O nível meso refere-se a estudos que exploram a práxis de estratégia nos níveis organizacional e intraorganizacional como um programa de mudança ou um processo estratégico. Por último, no nível macro se encontram os estudos que tentam explicar a prática sob a perspectiva do nível institucional, que frequentemente está relacionado com a explanação de padrões de ação de uma indústria específica.

\section{b) Práticas}

As práticas envolvem as várias rotinas, discursos, conceitos e tecnologias por meio das quais o trabalho estratégico se torna possível (JARZABKOWSKI; WHITTINGTON, 2008). Schatzki (2006) argumenta que uma organização é um conjunto de práticas e arranjos. Tal afirmação implica dizer que uma organização consiste em inter-relações entre práticas e ordens materiais. Assim, entende-se que as práticas são caracterizadas como ferramentas sociais, simbólicas e materiais por meio das quais a estratégia é realizada (JARZABKOWSKI; SPEE, 2009). 
De acordo com Jarzabkowski, Balogun e Seidl (2007), o uso das práticas está intrinsecamente relacionado ao strategizing porque elas fornecem os recursos comportamentais, cognitivos, processuais, discursivos e físicos por meio dos quais múltiplos atores são capazes de interagir para realizar socialmente a atividade coletiva. Além disso, os estudiosos de estratégia como prática percebem a natureza incorporada e institucionalizada das práticas que fornecem compreensão compartilhada de como fazer estratégia (JARZABKOWSKI, 2004; CHIA; HOLT, 2007; SEIDL 2007; JARZABKOWSKI; WHITTINGTON, 2008).

As práticas materiais de ferramentas como PowerPoint e outras tecnologias, as práticas existentes na lógica tácita do know-how sobre conceitos e discursos e os modos habituais de fazer estratégia, tais como revisões e reuniões são formas reconhecidas de fazer estratégia (JARZABKOWSKI; WHITTINGTON, 2008). Nessa perspectiva, são práticas: as formas de atividades corporais e mentais, as "coisas" e suas formas de utilização, o conhecimento passado na forma de compreensão, o saber como fazer, os estados de emoção e o conhecimento motivacional (RECKWITZ, 2002; JARZABKOWSKI; BALOGUN; SEIDL, 2007).

Apesar dessas definições e de haver uma agenda de pesquisa em evidência a respeito das práticas de estratégia, a literatura de estratégia como prática - em especial a literatura empírica - não apresenta uma visão dominante sobre o conceito (JARZABKOWSKI; WHITTINGTON, 2008). Dessa maneira, o estudo das práticas evidencia sua importância no processo de construção da realidade social e destaca as respostas acerca das questões: de onde vêm as atividades do strategizing? Como acontecem essas atividades? Quem são os responsáveis por efetivá-las? Quais as competências básicas para a execução das estratégias? É a consideração desses questionamentos que dão relevância à prática (ZWICK; SILVA; BRITO, 2014).

\section{c) Praticantes}

Os praticantes são indivíduos da estratégia que elaboram práticas, e, portanto, se relacionam com as práticas e práxis, sendo a conexão crítica entre esses dois elementos. Sob a perspectiva estratégica, os praticantes têm papel ativo na formação da organização e sua sobrevivência. O que esses atores realmente fazem é práxis estratégica, eles moldam a atividade estratégica por meio de quem eles são, como agem e quais práticas elaboram (WHITTINGTON, 2006; JARZABKOWSKI; BALOGUN; SEIDL, 2007).

Página 65 Caderno de Ciências Sociais Aplicadas, Vitória da Conquista/BA, vol. 15, nº 25, ano 15, p. 57-73, jan/jun 2018. 
Coraiola, Oliveira e Gonçalves (2012) encontraram, em uma revisão de literatura, quatro vertentes da estratégia como prática que tratam dos estrategistas (ou praticantes): foucaultiana, das representações sociais, da atividade e da estruturação. Entre as críticas encontradas a respeito desses estrategistas estão: (i) praticantes da estratégia são idealizados principalmente nas abordagens teóricas; (ii) frequentemente estrategistas são relacionados com pessoas, entidades individuais particulares; (iii) aqueles que realizam estratégias, as realizam de forma voluntária e possuem competência suficiente para tomar decisões; (iv) os agentes estratégicos são necessariamente aqueles pertencentes à diretoria da organização; e (v) atores não humanos e que não realizam a estratégia diretamente são desconsiderados na análise estratégica (CORAIOLA; OLIVEIRA; GONÇALVES, 2012).

Da ótica da estratégia como prática social, Jarzabkowski e Whittington (2008, 2009) argumentam que os praticantes da estratégia incluem aqueles que participam diretamente da elaboração da estratégia - como os gerentes e consultores - e os que influenciam indiretamente - como os decisores políticos e os meios de comunicação. Uma forma de classificar esses praticantes é defini-los como internos ou externos. Os praticantes internos são aqueles que possuem uma função dentro da organização, enquanto os externos, apesar de não terem uma hierarquia na estrutura da empresa, são capazes de influenciar a estratégia.

Outro ponto relevante quanto aos praticantes estratégicos é que eles não são necessariamente praticantes individuais, mas também podem ser grupos de pessoas (JARZABKOWSKI; SPEE, 2009).

\section{d) Strategizing}

A união entre praticantes, práxis e práticas é o cerne da pesquisa em estratégia como prática. Apesar de distintos, esses conceitos são interconectados de forma que não é possível estudar um sem também considerar os aspectos dos outros (JARZABKOWSKI; BALOGUN; SEIDL, 2007). A polissemia e interconexão de conceitos fazem parte do contexto do entendimento da estratégia como uma prática social, visto que os significados daí obtidos são utilizados nas atividades de strategižing (RESE et al., 2017). Essa união é o strategizing, isto é, o processo de fazer estratégia. Na Figura 2 é possível observar um modelo estrutural para análise da estratégia como prática social.

Página 66 Caderno de Ciências Sociais Aplicadas, Vitória da Conquista/BA, vol. 15, nº 25, ano 15, p. 57-73, jan/jun 2018. 


\section{Cadernos de CiênCIas SOCIAIS APLICADAS}

O strategiz̨ing abrange as ações, negociações e interações dos diversos praticantes e as práticas dos mesmos para realizar essas atividades. Significa "fazer estratégia", processo em que são construídas as interações entre a práxis, a prática e os praticantes (JARZABKOWSKI; BALOGUN; SEIDL, 2007; WALTER; AUGUSTO, 2012).

Figura 2: Estrutura conceitual para analisar a estratégia como prática.

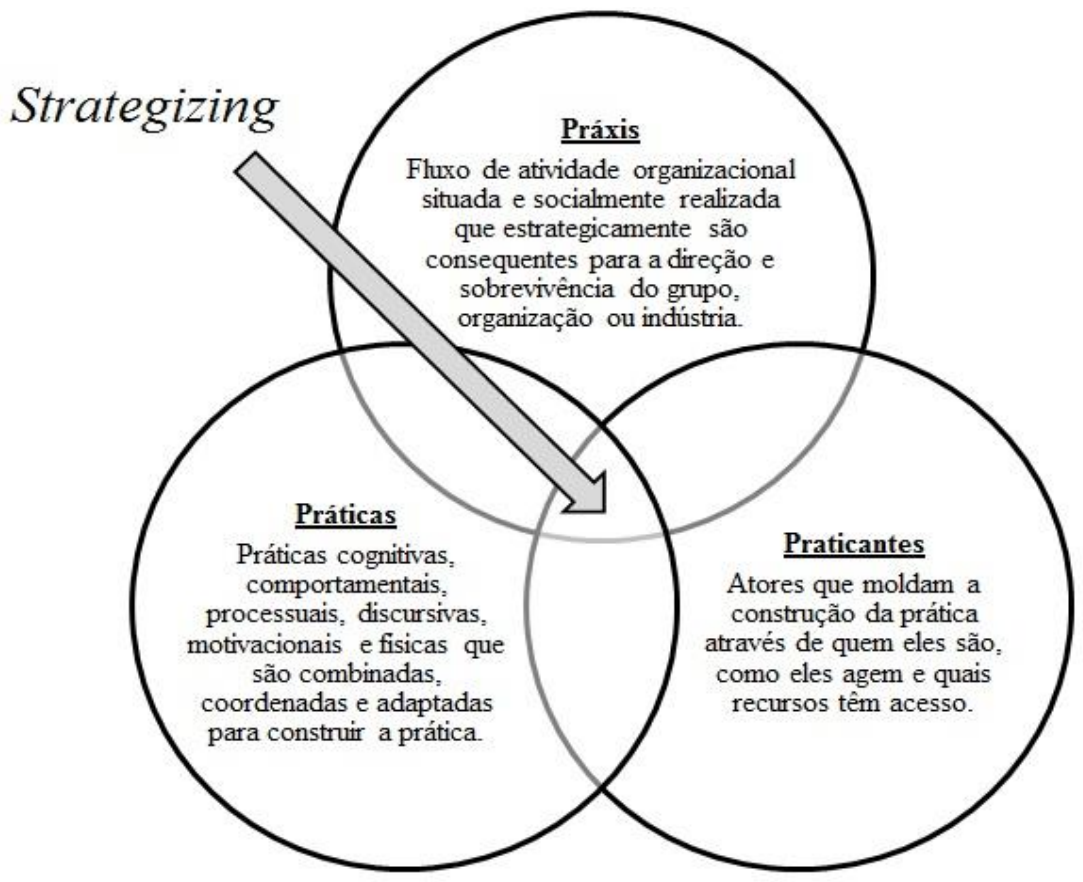

Fonte: Adaptado de Jarzabkowski; Balogun; Seidl (2007, p. 11).

A noção de fazer estratégia também compreende a interação entre o pensar e o agir estrategicamente. Nesse sentido, é abandonada a ideia de que a estratégia consiste em um grande período de formulação - em que os estrategistas pensam a respeito da estratégia -, seguida por outro grande período de implementação da estratégia - período de ação dos estrategistas. Pela lógica do strategižing, o pensar e o agir estrategicamente estão fortemente conectados e se sustentam mutuamente, não sendo necessária uma interação contínua nem linear (WILSON; JARZABKOWSKI, 2004).

Página 67 Caderno de Ciências Sociais Aplicadas, Vitória da Conquista/BA, vol. 15, nº 25, ano 15, p. 57-73, jan/jun 2018. 


\section{Considerações finais}

O debate epistemológico gerado pelos estudos a respeito da estratégia como prática social tem contribuído para os estudos organizacionais e, em especial, para o campo de estudos da estratégia (ZWICK; SILVA; BRITO, 2014). O objetivo desse artigo foi oferecer uma sistematização das definições e conceitos da proposta de Estratégia como Prática e, para isso, foram trazidos os argumentos dos principais autores que têm estudado a temática.

Desde o surgimento do campo de estudos da estratégia, diversos significados puderam ser atribuídos a ela (MINTZBERG, 2006), todos eles de certa forma relevantes para orientar a prática organizacional (MINTZBERG; AHLSTRAND; LAMPEL, 2000). A estratégia como prática, no entanto, teve maior destaque a partir do artigo publicado em 1996 por Whittington, "Strategy as practice", em que o autor define quatro perspectivas de estratégia: política, planejamento, processo e prática.

$\mathrm{Na}$ concepção de estratégia como prática social, o trabalho estratégico vai além da formulação, mas também envolve os cuidados compreendidos na implementação e todas as atividades que contribuem para o surgimento de estratégias organizacionais. Sendo assim, a estratégia se trata de uma prática social, uma atividade socialmente realizada devido às relações de diferentes praticantes (JARZABKOWSKI; SPEE, 2009).

As pesquisas em estratégia como prática consideram que a vida social se dá dentro das práticas e que as práticas e os praticantes são fundamentais para esse tipo de estudo. É uma abordagem intepretativista em que o mundo, os atores sociais e os processos que o produzem encontram-se totalmente vinculados (JARZABKOWSKI; KAPLAN, 2010).

A estratégia como prática, portanto, é uma alternativa às perspectivas que consideram somente o nível macro e desvalorizam as relações entre indivíduos e as práticas organizacionais (TURETA; LIMA, 2011). Nesse tipo de estudo, o pesquisador busca compreender mais que as simples ações das pessoas, investigando também como e por que tais ações ocorrem e atribuindo à estratégia um conjunto de atividades em transformação contínua (TURETA; LIMA, 2011). A questão-chave a ser considerada nesse tipo de investigação é a reflexividade, isto é, refletir sobre os fenômenos facilitadores e inibidores das práticas sociais e dedicar atenção especial àquilo que é tido como certo por acadêmicos e não acadêmicos (VAARA; WHITTINGTON, 2012).

Por fim, Jarzabkowski, Balogun e Seidl (2007) argumentam que a estratégia como prática, em concordância com outras teorias organizacionais, baseia-se, entre outros, nos parâmetros meta-teóricos

Página 68 Caderno de Ciências Sociais Aplicadas, Vitória da Conquista/BA, vol. 15, n 25, ano 15, p. 57-73, jan/jun 2018. 
da sociologia, da psicologia social, da antropologia e da etnometodologia para compreender a construção da atividade dentro das organizações (JARZABKOWSKI; BALOGUN; SEIDL, 2007). O campo de pesquisa da estratégia como prática enriquece os estudos relacionados à estratégia tradicional de quatro formas: (i) aborda primordialmente as teorias sociológicas da prática, em vez das teorias de cunho econômico; (ii) amplia o alcance do que a pesquisa em estratégia pode explicar; (iii) amplia significativamente o âmbito setorial da pesquisa em gestão estratégica, levando reflexões para além da lógica empresarial com fins lucrativos; (iv) possibilita uma significativa mudança metodológica (VAARA; WHITTINGTON, 2012).

Quanto aos cuidados que os estudiosos devem ter ao pesquisar a estratégia como uma prática social, destacam-se os seguintes: em primeiro lugar, é importante que o contexto em que as práticas são realizadas também seja analisado, pois é esse contexto/sociedade que define as práticas que moldam e permitem a atividade humana (WHITTINGTON, 2006). Faz-se necessário também enxergar além do que simplesmente é feito na prática. A atividade real da pessoa "na prática" demanda atenção especial em relação a como as coisas são feitas (WHITTINGTON, 2006). Os atores/estrategistas/praticantes também possuem papel fundamental nos estudos de estratégia como prática social. Eles têm a habilidade de fazer a diferença uma vez que podem modificar ou repetir práticas anteriores (WHITTINGTON, 2006). Por último, mesmo diante dos desafios e dificuldades de publicações desse tipo de pesquisa - devido a fatores como crítica ao método qualitativo empregado nos estudos e até mesmo resistência a esse significativamente "novo" campo, bem como a dificuldade dos revisores de periódicos em aceitar o termo strategiz̨ing - as recompensas para estudos nessa área são grandes, pois a estratégia como prática pode propiciar conhecimentos mais abrangentes do que o oferecido geralmente pela gestão estratégica (JARZABKOWSKI; KAPLAN, 2010).

A contribuição do presente artigo consiste em revisar as definições e conceitos relacionados aos estudos acerca da Estratégia como Prática Social. Uma vez que se trata de um campo de pesquisa em crescimento, uma sistematização das discussões realizadas até o momento se faz necessária para auxiliar no entendimento do tópico, bem como no direcionamento para futuras investigações.

Esse ensaio não esgota a literatura sobre estratégia como prática, e, portanto, pesquisas posteriores podem desenvolver estudos de cunho bibliométrico, trazendo informações mais precisas sobre a realidade dos trabalhos no campo. Incentiva-se também a produção de pesquisas científicas empíricas sobre o tema, devido as lacunas percebidas no decorrer do estudo aqui realizado.

Página 69 Caderno de Ciências Sociais Aplicadas, Vitória da Conquista/BA, vol. 15, n 25, ano 15, p. 57-73, jan/jun 2018. 


\section{Referências}

ANDRIGUETTOO JUNIOR, Haroldo. A contribuição da estratégia como prática para o estudo da formação de estratégias em universidades. RPGE- Revista on line de Política e Gestão Educacional, v.20, n.2, p. 337-358, 2016.

BOURDIEU, Pierre. The logic of practice. Stanford University Press, 1990.

CAMARGOS, Marcos Antonio; DIAS, Alexandre Teixeira. Estratégia, administração estratégica e estratégia corporativa: uma síntese teórica. Caderno de Pesquisas em Administração, v. 10, n. 1, p. 27-39, 2010.

CHANDLER, Alfred Dupont. Strategy and Structure: chapters in the History of the American Industrial Enterprise. Cambridge: The MIT Press. 1962.

CHIA, Robert; HOLT, Robin. Strategy as practical coping: A Heideggerian perspective. Organization Studies, v. 27, n. 5, p. 635-655, 2006.

CORAIOLA, Diego Maganhotto; OLIVEIRA, Samir Adamoglu; GONÇALVES, Sandro Aparecido. Se a estratégia é prática, quem são seus praticantes?. Revista Brasileira de Estratégia, Curitiba, v. 5, n. 3, p. 231-242, 2012.

DE CERTEAU, Michel. A invenção do cotidiano: artes de fazer. Petrópolis: Vozes. 1984.

DIAS, Almerinda Tereza Bianca Bez Batti; ROSSETTO, Carlos Ricardo; MARINHO, Sidnei Vieira. Estratégia como Prática Social: um Estudo de Práticas Discursivas no Fazer Estratégia. Revista de Administração Contemporânea, v. 21, n. 3, p. 393-412, 2017.

DOXSEY Jaime Roy; DE RIZ, Joelma. Metodologia da pesquisa científica. ESAB - Escola Superior Aberta do Brasil, 2003. Apostila.

GIDDENS, Anthony. The constitution of society. Cambridge: Polity Press, 1984.

JARZABKOWSKI, Paula; WHITTINGTON, Richard. Hard to disagree, mostly. Strategic Organization, v. 6, n. 1, p. 101-106, 2008.

Página 70 Caderno de Ciências Sociais Aplicadas, Vitória da Conquista/BA, vol. 15, n 25, ano 15, p. 57-73, jan/jun 2018. 


\section{Cadernos de CiênCIas SOCIAIS APLICADAS}

JARZABKOWSKI, Paula. Strategy as practice: recursiveness, adaptation, and practices-in-use. Organization Studies, v. 25, n. 4, p. 529-560, 2004.

JARZABKOWSKI, Paula. Strategy as practice: An activity based approach. London: Sage, 2005.

JARZABKOWSKI, Paula; BALOGUN, Julia; SEIDL, David. Strategizing: The challenges of a practice perspective. Human Relations, v. 60, n. 1, p. 5-27, 2007.

JARZABKOWSKI, Paula; KAPLAN, Sarah. Taking "strategy-as-practice" across the Atlantic. In: BAUM, Joel A. C.; LAMPEL, Joseph. Advances in Strategy Management - Volume 27: The Globalization of Strategy Research. Emerald Group Publishing Limited, 2010. p. 51-71.

JARZABKOWSKI, Paula; SPEE, Andreas Paul. Strategy-as-practice: A review and future directions for the field. International Journal of Management Reviews, v. 11, n. 1, p. 69-95, 2009.

JARZABKOWSKI, Paula; SPEE, Andreas Paul; SMETS, Michael. Material artifacts: Practices for doing strategy with 'stuff. European Management Journal, v. 31, n. 1, p. 41-54, 2013.

MACIEL, Cristiano de Oliveira; AUGUSTO, Paulo Otavio Mussi. A practice turn e o movimento social da estratégia como prática: está completa essa virada?. RAM, v. 14, n. 2, p. 155-178, 2013.

MARITZ, Rachel; DU TOIT, Adeline. The practice turn within strategy: Competitive intelligence as integrating practice. South African Journal of Economic and Management Sciences, v. 21, n. 1, p. 14, 2018.

MENEGHETTI, Francis Kanashiro. O que é um ensaio-teórico?. RAC-Revista de Administração Contemporânea, v. 15, n. 2, p. 320-332, 2011.

MINTZBERG, Henry; AHLSTRAND, Bruce; LAMPEL, Joseph. Safári de estratégia. Porto Alegre: Bookman. 2007.

NICOLAU, Isabel. O conceito de estratégia. INDEG/ISCTE, 2001.

PORTER, Michel E. O que é estratégia. Harvard Business Review, v. 74, n. 6, p. 61-78, 1996.

Página 71 Caderno de Ciências Sociais Aplicadas, Vitória da Conquista/BA, vol. 15, n 25, ano 15, p. 57-73, jan/jun 2018. 


\section{Cadernos de CiênCIas SOCIAIS APLICADAS}

RECKWITZ, Andreas. Toward a theory of social practices: A development in culturalist theorizing. European Journal of Social Theory, v. 5, n. 2, p. 243-263, 2002.

RESE, Natália, KUABARA, Flávia Harumi Souza, VILLAR, Eduardo Guedes, FERREIRA, Jane Mendes. O Vir a Ser da Estratégia como uma Prática Social. RAC, v. 21, n. 2, p. 227-248, 2017.

RONDA-PUPO, Guillermo Armando; GUERRAS-MARTIN, Luis Ángel. Dynamics of the evolution of the strategy concept 1962-2008: a co-word analysis. Strategic Management Journal, v. 33, n. 2, p. 162-188, 2012.

SAMPAIO, Isabel Cristina; FORTUNATO, Graziela; BASTOS, Sergio Augusto Pereira. A estratégia como prática social: o pensar e o agir em um programa social governamental. O\&S, v. 20, n. 66, p. 479499, 2013.

SCHATZKI, Theodore R. Social practices: A Wittgensteinian approach to human activity and the social. Cambrigde: Cambridge University Press, 1996.

SCHATZKI, Theodore R. On organizations as they happen. Organization Studies, v. 27, n. 12, p. 1863-1873, 2006.

SEIDL, David. General strategy concepts and the ecology of strategy discourses: A systemic-discursive perspective. Organization Studies, v. 28, n. 2, p. 197-218, 2007.

TURETA, César; LIMA, Juvencio Braga. Estratégia como prática social: o estrategizar em uma rede interorganizacional. RAM, v. 12, n. 6, p. 76-108, 2011.

VAARA, Eero; WHITTINGTON, Richard. Strategy-as-practice: taking social practices seriously. Academy of Management Annals, v. 6, n. 1, p. 285-336, 2012.

WALTER, Silvana Anita; AUGUSTO, Paulo Otávio Mussi. Prática estratégica e strategizing: mapeamento dos delineamentos metodológicos empregados em estratégia como prática. RECADM, v. 11, n. 1, p. 131-142, 2012.

Página 72 Caderno de Ciências Sociais Aplicadas, Vitória da Conquista/BA, vol. 15, nº 25, ano 15, p. 57-73, jan/jun 2018. 


\section{- CADERNos de CIIENCIAS SOCIAIS APLICADAS}

WALTER, Silvana Anita; AUGUSTO, Paulo Otávio Mussi; FONSECA, Valéria Silva. O campo organizacional e a adoção de práticas estratégicas: revisitando o modelo de Whittington. Cadernos EBAPE.BR, v. 9, n. 2, p. 282-298, 2011.

WHITTINGTON, Richard. Strategy as practice. Long Range Planning, v. 29, n. 5, p. 731-735, 1996. WHITTINGTON, Richard. Completing the practice turn in strategy research. Organization Studies, v. 27 , n. 5, p. 613-634, 2006.

JARZABKOWSKI, Paula; WILSON, David C. Pensando e agindo estrategicamente: novos desafios para a análise estratégica. RAE, v. 44, n. 4, p. 11-20, 2004.

ZWICK, Elisa; SILVA, Isabel Cristina; BRITO, Mozar José. Estratégia como prática social e teoria da ação comunicativa: possíveis aproximações teóricas. Cadernos EBAPE.BR, v. 12, p. 384-400, 2014. 